\title{
Fat embolism syndrome
}

\author{
Guruprasad Sogunuru $\cdot$ Nagabhishek Moka
}

Received: 29 August 2009 / Accepted: 11 January 2010/Published online: 1 April 2010

(C) Springer-Verlag London Ltd 2010

A 40-year-old man who sustained polytrauma presented with comminuted fractures of both right tibia and femur to the emergency room (ER). He had no evidence of head injury and was admitted for surgical fixation. Twelve hours after admission he developed dyspnea and while awaiting surgery his sensorium gradually declined over the next $24 \mathrm{~h}$. Examination revealed a pulse rate of $130 \mathrm{bpm}$, blood pressure of 120/82 $\mathrm{mmHg}$, respiratory rate of 32 breaths $/ \mathrm{min}$, and oxygen saturation of $85 \%$. On general examination petechiae were noted in palpebral conjunctiva and in axillae. Auscultation revealed normal heart sounds and fine diffuse inspiratory crackles. The clinical possibilities considered were: fat embolism, pulmonary thromboembolism, traumatic pneumothorax, and acute respiratory distress syndrome. The presence of normal vesicular breath sounds and normal chest $\mathrm{X}$-ray made the possibilities of pneumothorax and acute respiratory distress syndrome unlikely. The short duration of the event and lack of predisposing conditions made pulmonary embolism due to venous thrombi unlikely. Hence the most probable clinical diagnosis was fat embolism syndrome. Other supportive evidence for this included: low hemoglobin (8.2 $\mathrm{g} \%$ ), thrombocytopenia (platelets 89,000/ $\mathrm{mm}^{3}$ ), and greasy urine (lipiduria). ECG revealed sinus tachycardia. The patient was managed conservatively till the

\footnotetext{
G. Sogunuru

c/o DBR Hospitals, Mosque Road,

Tirupathi, India

e-mail: drguru73@yahoo.com

N. Moka $(\bowtie)$

c/o SVRRGGH,

Tirupathi, India

e-mail: nagabhishek@yahoo.com
}

surgery. Fat embolism (FE) develops in $90 \%$ of all bone fractures. Fat embolism syndrome (FES) is a serious manifestation of FE with an incidence of $0.5-2.0 \%$ among all long bone fractures. Usually it manifests within $72 \mathrm{~h}$ of initial insult. This patient showed the classic triad of FES consisting of hypoxemia, neurological abnormalities, and petechial rash. The characteristic petechial rash is seen in $30-50 \%$ of patients on head and neck, anterior thorax, subconjunctiva, and axilla. The exact pathogenesis is unclear but includes mechanical obstruction and biochemical injury. Clinical diagnosis is the key because lab and imaging modalities (high-resolution computed tomography) are not specific. Management is primarily supportive including assisted ventilation and intensive care unit (ICU) monitoring; the role of steroids is still controversial.
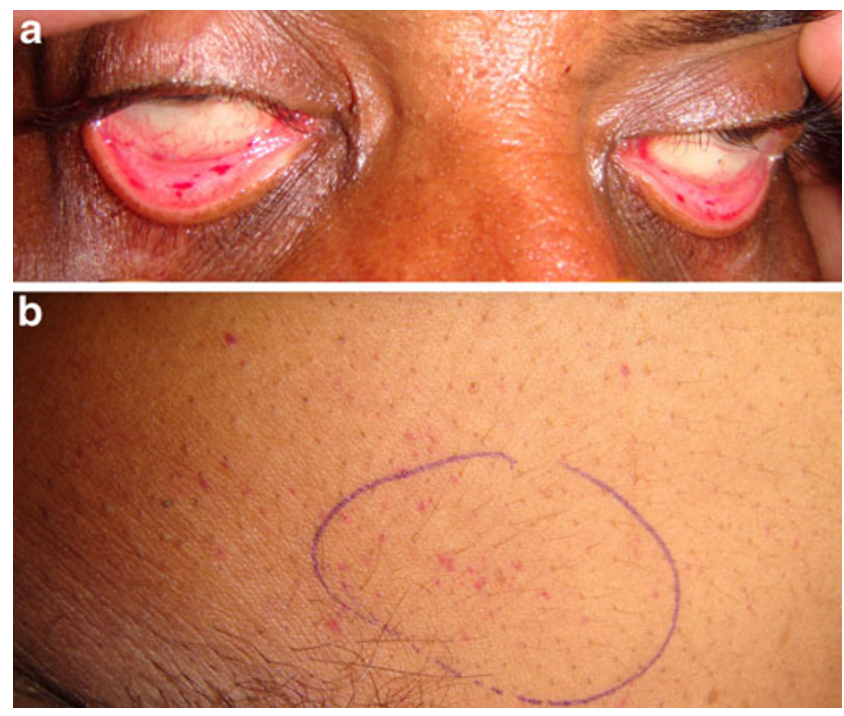

Fig. 1 a Lower palpebral conjunctivae showing petechiae. b Multiple petechiae in the right axilla 